\title{
Spindles take charge
}

Non-rapid eye movement (NREM) sleep is characterized by three patterns of rhythmic brain activity: hippocampal sharp-wave ripples, thalamic spindles and slow cortical oscillations. Phase-locked interactions between these rhythms have been demonstrated and are proposed to be crucial for the memorypromoting effects of NREM sleep; however, the specific contribution of thalamic spindles to this process is not known. Latchoumane et al. now show that, in mice, thalamic spindles contribute to the triple coupling of ripples, spindles and slow oscillations during NREM sleep, a process that is likely to be a central mechanism supporting memory formation during sleep.

The consolidation of hippocampaldependent memories is thought to be a multi-step process in which information is transferred from the hippocampus to cortical networks for long-term storage. To investigate the contribution of thalamic spindle activity to memory consolidation, the authors used an optogenetic approach to stimulate spindle-like activity in the thalamic reticular nucleus (TRN) of mice during NREM sleep after training in a contextual fear conditioning task. By simultaneously recording electroencephalographic information from the cortex, they established a closed-loop stimulation approach that ensured that the stimulated spindles coincided with particular phases of the ongoing slow cortical oscillations.

Using this approach, the authors found that spindle stimulation that occurred in phase (that is, coupled to the excitable phase of the slow oscillation, the so-called 'up state') enhanced fear memory consolidation, whereas stimulation that occurred out of phase with respect to the slow oscillation up state did not have this effect. Similarly, optogenetic inhibition of in-phase spindle activity impaired memory consolidation, whereas inhibition of out-of-phase spindles had no effect. Thus, only thalamic spindles that are phase coupled to the up state of the slow oscillation can support memory consolidation.

This experimental evidence for the importance of the phase coupling between the two different brain rhythms in memory consolidation prompted the authors to consider how the third rhythm, hippocampal ripples, is coordinated. Local field potential recordings showed that in-phase spindle stimulation was able to strengthen the 'nesting' of hippocampal ripples within the excitable phases (troughs) of the cycles of spindles recorded in the hippocampus or parietal cortex. Furthermore, the in-phase stimulation was able to increase the incidence of 'triple-coupled' slow oscillations, spindles and ripples, indicating a central role for thalamic spindles in coordinating these three brain rhythms.

Although the precise mechanisms that mediate the phase coupling of hippocampal, thalamic and cortical activity during sleep remain unknown, this study suggests that thalamic spindle activity has a crucial organizing role in this process and is essential for memory consolidation.

Katherine Whalley

ORIGINAL ARTICLE Latchoumane, C-F. V. et al. Thalamic spindles promote memory formation during sleep through triple phase-locking of cortical, thalamic, and hippocampal rhythms. Neuron http://dx.doi.org/10.1016/j. neuron.2017.06.025 (2017)

\section{$c 6$}

in-phase

stimulation

was able to

increase the

incidence

of 'triple-

coupled' slow

oscillations,

spindles and

ripples

g

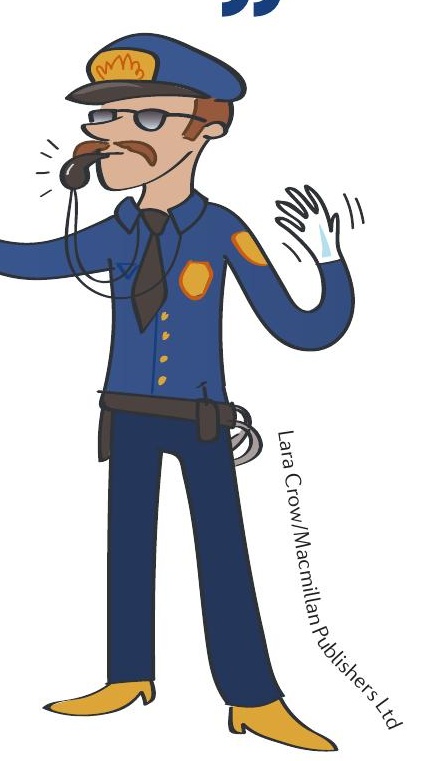

